La Costituzione dello stato di Puebla fra elementi di originalità e influenze federali*

\title{
Eleonora CECCHERINI**
}

SOMMARIO: I. Alcune considerazioni generali sul testo. II. I profili di originalità. III. I diritti delle popolazioni indigene fra garanzie e inattuazione. IV. Alcune note conclusive.

\section{ALCUNE CONSIDERAZIONI GENERALI SUL TESTO}

Il contributo vuole dare conto di alcuni profili emergenti nel diritto costituzionale degli stati membri della Repubblica degli Stati Uniti di Messico, partendo dall'analisi della costituzione di Puebla. Il suo testo è stato approvato dal Congresso statale, 1'8 settembre 1917 ed è entrato in vigore il 1o. ottobre dello stesso anno. Era stato il decreto federale numero 13 del 1917 a imporre a tutti gli stati della Federazione l'indizione di nuove elezioni per le assemblee locali, che avevano il compito specifico di approvare la costituzione.

In primo luogo, pare utile inquadrare tale atto nel sistema delle fonti: la costituzione federale definisce all'art. 40 gli stati membri come sovrani, tuttavia, non possono vantare questa caratteristica che sarebbe di pertinenza esclusiva della Federazione. Non sembra neppure che si sia imposta l'idea di una sovranità condivisa fra Federazione ed entità federative, tant'è

* Si ringraziano il professore Pedro Salazar Ugarte, direttore dell'Instituto de Investigaciones Jurídicas dell'Universidad Nacional Autónoma de México e i suoi collaboratori per avermi fornito alcuni testi per la stesura di questo contributo.

** Universidad de Genova, Italia, Contatto: eleonora.ceccherini@unige.it; https:// orcid.org/0000-0002-4291-5730. 
che il Plenum della Suprema Corte di Giustizia ha dichiarato che queste ultime sono parti costitutive dell'Unione messicana ma soggette all'imperio della costituzione federale e, quindi, prive di poteri sovrani; a loro sarebbero infatti riconducibili solo sfere di autonomie costituzionalmente garantite. ${ }^{1}$ In questa ottica, si iscrive l'art. 41 della costituzione, che esplicita la clausola di supremazia della carta fondamentale federale.

Tale disposto ha finito per configurarsi de facto, prima ancora che de jure, come un obbligo per le costituzioni statali di recepire testualmente le revisioni della carta fondamentale federale. Questo "gioco di specchi" spiega le ragioni per cui le disposizioni della costituzione di Puebla riproducono in maniera quasi anastatica le prescrizioni della Grundnorm federale. ${ }^{2}$ Un esempio particolarmente calzante è l'art. 20, par. V che afferma che i ministri di culto dispongono dei diritti che la costituzione degli stati uniti messicani, le leggi e i regolamenti federali riconoscono loro. Come è evidente, si tratta di una disposizione che opera un semplice richiamo alle fonti di ambito federale, la cui vincolatività nel territorio di Puebla non de-

1 Amparo en revisión 5261/76 Gas Hatusco, S. de R.L. 7 agosto 1979; Martí Capitanachi, Luz del Carmen, Las Constituciones locales en el sistema federal mexicano ¿Son verdaderas Constituciones?, https://biblio.juridicas.unam.mx/bjv (2002); Enríquez Soto, Pedro Antonio, "Régimen constitucional de las entidades federativas", www.juridicas.unam.mx. La distinzione è nota nel diritto comparato ed è resa nota già da Kelsen, Hans, Teoria generale del diritto e dello Stato, Milano, Comunità, 1950 (rist. Milano, Estaslibri, 1984), pp. 322 e ss; e nella dottrina italiana: cfr., De Vergottini, Giuseppe, "Stato federale", Enciclopedia del diritto, vol. XLIII, 1990, pp. 831 e ss. Sul tema delle trasformazioni del federalismo, Morrone, Andrea, Federalismo y democracia, relazione presentata il 6 gennaio 2018 al Seminario di Bologna, gentilmente concesso dall'autore; Rolla, Giancarlo, L'autonomia delle comunità territoriali, Milano, Giuffrè, 2008, p. 8. Più specificamente sul federalismo messicano: Luatti, Lorenzo, "Il federalismo «virtuale» latinoamericano. Vicende storiche, problemi e prospettive”, in Groppi, Tania (a cura di), Principio di autonomia e forma di Stato, Torino, Giappichelli, 1998, pp. 361 ss.; Valadés, Diego e Carpizo, Jorge, "Problemas del federalismo mexicano", in Trujillo, Gumersindo (coord.), Federalismo y regionalismo, Madrid, CEC, 1979, p. 197; Gutiérrez Parada, Oscar, Sistema federal, Ciudad de México, Porrúa, 2016, pp. 175 e ss.; Laynez Potisak, Javier, "Facultades concurrentes y federalismo", in Esquivel, Gerardo e Ibarra Palafox, Francisco Alberto e Salazar Ugarte, Pedro (coords.), Cien ensayos sobre el centenario de la Constitución Política de los Estados Unidos de México. Estudios jurídicos, vol. II, México, UNAM, Instituto de Investigación Jurídica, 2017, p. 271.

2 Sul punto Cossío, José Ramón, "La influencia de la Constitución española de 1978 en las Constituciones locales mexicanas", in Fernández Segado, Francisco (coord.), La Constitución de 1978 y el constitucionalismo iberoamericano, Madrid, CEC, 2003, pp. 520 e ss.

Esta obra está bajo una Licencia Creative Commons

Atribución-NoComercial-SinDerivar 4.0 Internacional, IIJ-UNAM. 
riva certamente dal loro inserimento nella Costituzione statale, non introducendo alcun elemento aggiuntivo alla condizione dei ministri di culto.

Peraltro, questa tecnica adeguatrice innesca una spirale che ha delle conseguenze non del tutto positive sulla sistematica del testo costituzionale statale. Infatti, poiché le riforme costituzionali federali sono state cospicue dal 1917 a oggi, queste hanno obbligato il legislatore di revisione statale a intervenire in maniera costante, incidendo più volte sul testo, compromettendone la sua organicità.

É percepibile una sorta di stratificazione costituzionale che rende meno intellegibile la ricostruzione dell'ordinamento costituzionale poblano. Questa carenza si riscontra, soprattutto, nell'ultima parte del testo, dove ad esempio, sono stati inseriti alcuni capitoli, dopo quelli relativi all'organizzazione dei pubblici poteri, che riguardano argomenti come l'istruzione pubblica (artt. 118, 119) e il diritto a un ambiente salubre (art. 121), anche se questi temi erano già stati anticipati nella parte relativa alla garanzia dei diritti.

Anche in base a questi rilievi, possiamo qualificare la Costituzione di Puebla come lunga. Si tratta, inoltre, di un testo in alcune parti estremamente specifico e non sempre - come abbiamo già evidenziato - sistematicamente ordinato.

Volendo argomentare le affermazioni precedenti e partendo dalla prima, merita precisare che la lunghezza non è causata tanto dal numero degli articoli - che sono 143 - quanto dalla loro suddivisione in paragrafi e frazioni, che rende ciascun articolo estremamente dilatato. D'altra parte, però, questo elemento colpisce forse chi è meno avvezzo alle tecniche di redazione costituzionale messicane, in quanto la stessa caratteristica si ripropone nella Costituzione federale, forse addirittura in maniera amplificata.

Questa particolare conformazione rende la lettura un po' più faticosa a un lettore meno attento e ciò sembra in contrapposizione a quanto viene solitamente affermato per spiegare la ragione di testi costituzionali estremamente prolissi, specifici e dettagliati. Proprio nella dottrina latinoamericana, si è fatta strada la posizione che carte fondamentali estremamente diffuse da un punto di vista stilistico assolverebbero a una funzione didascalica e informativa, soprattutto per una cittadinanza non sempre pienamente consapevole dei propri diritti e dell'organizzazione dei pubblici poteri. 
Tuttavia, 1"“ipertestualità" con funzioni di tipo civico, in genere è associata al catalogo dei diritti, al contrario, analizzando il testo emerge un profilo di segno opposto e cioè che la Costituzione statale non si è arricchita di un corposo bill of rights, neppure nella forma più debole di norme manifesto. $^{3}$

Tale aspetto richiama l'attenzione in quanto nel diritto comparato i testi fondamentali delle entità substatali integrano generalmente cataloghi dei diritti, anzi, questo sembra essere l'aspirazione più diffusa. La spiegazione risiede nella volontà dei legislatori locali di garantire un certo numero di diritti che incarnino l'idea di una sorta di cittadinanza decentrata. ${ }^{4} \mathrm{Nel}$ testo si vogliono proiettare interessi, diritti e aspirazioni della comunità. Tuttavia, la carta fondamentale di Puebla non sembra assecondare questa tendenza e annovera al suo interno solo alcune indicazioni, seppur non prive di specifico interesse, che sono riconducibili ai diritti sociali e a nuovi diritti, ma che, comunque, sono mutuate dalla costituzione federale. ${ }^{5} \mathrm{~A}$ titolo esemplificativo, vediamo che nel capitolo III, rubricato come "diritti umani e sue garanzie", si rinviene un obbligo per il legislatore ad assicurare il benessere dei minori, provvedendo alle loro necessità in materia di istruzione e alimentazione. Il riferimento al tema dei bisogni alimentari

3 Questo assunto sembra abbastanza consolidato nel diritto comparato, fin dal 1990 nel Working Document del National Congress in Sudafrica si affermava a proposito degli obiettivi di un testo costituzionale: "Moreover, it terms should be such tha any person can understand them", la citazione è tratta da Chanock, Martin, "A Post-Calvinist Catechism or a Communist Manifesto? Intersecting Narratives in the South African Bill of Rights Debate", in Alston, Philip (ed.), Promoting Human Rights Through Bills of Rights, Oxford, Oxford University Press, 1999, p. 412.

4 A titolo esemplificativo, possiamo citare l'esperienza statutaria italiana post riforma del 2001, in cui gli Statuti delle regioni ordinarie hanno proceduto a introdurre molti diritti nei loro testi. Sul tema, la bibliografia è corposa e senza pretesa di esaustività, si rimanda a: Gambino, Silvio, "Ordinamento regionale e diritti fondamentali", in Gambino, Silvio (a cura di), Diritto regionale, Milano, Giuffrè, 2009, pp. 141 e ss.; Longo, Erik, "Il ruolo dei diritti negli statuti e nelle istituzioni regionali", in Di Cosimo, Giovanni (a cura di), Statuti Atto II. Le regioni e la nuova stagione statutaria, Macerata, Eum, 2007, pp. 41 e ss.; Caretti, Paolo, "La disciplina dei diritti fondamentali è materia riservata alla Costituzione”, Le regioni, 2005, pp. 27 e ss.; Flick, Giovanni Maria, "Nuovi diritti fondamentali e ruolo delle regioni”, Quaderni regionali, num. 1, 2009, pp. 9 e ss.; Adamo, Ugo, La questione dei principi e diritti negli statuti delle regioni e delle comunità autonome, Milano, Giuffrè, 2015.

5 Sánchez Cordero Dávila, Olga María del Carmen, "Los efectos de la reforma en derechos humanos en el constitucionalismo local", in Esquivel, Gerardo e Ibarra Palafox, Francisco Alberto e Salazar Ugarte, Pedro (coords.), op. cit., p. 413.

Esta obra está bajo una Licencia Creative Commons

Atribución-NoComercial-SinDerivar 4.0 Internacional, IIJ-UNAM. 
è sicuramente suggestivo e costituisce quasi una "nuova frontiera" per il diritto costituzionale,${ }^{6}$ ma anche di questa esigenza si ha eco nella Costituzione federale all'art. $4 \mathrm{o}$.

\section{I PROFILI DI ORIGINALITÀ}

Alcuni riflessi di originalità, invece, emergono nel titolo I, capitolo V, artt. 26 e 27 dedicati alla famiglia ovvero nel titolo VIII capitolo V, art. 121 sul diritto all'ambiente. Volgendo lo sguardo al primo tema, si evince che non è uno fra quelli arati nel testo federale, ${ }^{7}$ mentre, al contrario, quello statale sembra attribuire alla comunità familiare una significativa rilevanza. Infatti, viene definita una istituzione fondamentale (art. 26), atta a promuovere l'insegnamento e la trasmissione dei valori culturali, etici e sociali necessari per lo sviluppo delle persone che la compongono. A questa disposizione, se ne aggiungono altre che hanno come oggetto di regolazione: il patrimonio familiare, le politiche familiari che, in particolare, assicurino protezione ai minori, ai disabili e agli anziani e il riconoscimento del pari valore del lavoro del padre e della madre all'interno della famiglia. Sono, inoltre, ribaditi i principi di eguaglianza, di solidarietà (che si traducono nell'obbligo di ciascun membro della famiglia a contribuire ai bisogni della stessa) e di rispetto reciproco. Infine, ampio spazio è riservato al tema dei figli, che, a prescindere dal sesso, sono eguali davanti alla legge e che i genitori hanno l'obbligo di istruire, educare, mantenere e assistere e, specularmente, la prole ha gli stessi doveri di assistenza nei confronti degli ascendenti, qualora siano impossibilitati a provvedere a se stessi. Sempre su questo tema, pare interessante il richiamo al dovere per i componenti del nucleo familiare di astenersi dal porre in essere condotte che possono generare violenza familiare e, pur essendo una norma manifesto, priva di un contenuto precettivo in senso stretto, non è da sottovalutarne l'impatto altamente simbolico.

\footnotetext{
6 Sul tema si vedano le considerazioni di Morrone, Andrea, "Ipotesi per un diritto costituzionale all'alimentazione", in Cerrina Feroni, Ginevra e Frosini, Tommaso Edoardo e Mezzetti, Luca e Petrillo, Pier Luigi (a cura di), Ambiente, energia, alimentazione. Modelli giuridici comparati per lo sviluppo sostenibile, cesinfin online, 2016, pp. 31 e ss.

7 Anche se non mancano per esempio riferimenti alla protezione della famiglia come limite di legittimità ai decreti adottati in stato di emergenza (art. 29) o alla stabilità familiare in relazione al diritto di amparo (art. 107, par. III, frac. a).
} 
Sempre in tema di famiglia si può notare un altro elemento che riguarda le clausole interpretative. Infatti, si richiamano i pubblici poteri a garantire lo sviluppo della famiglia in ossequio - non solo alla Costituzione federale e alle fonti pattizie - ma a tutti gli strumenti internazionali ratificati dallo Stato messicano (art. 26). ${ }^{8}$ Sembra, quindi, che il tema della famiglia rivesta una particolare e specifica rilevanza, infatti, vi si dedica una disposizione speciale, che sembra avere un contenuto più ampio della clausola generale sulla gerarchia delle fonti della Costituzione federale (art. 10) ${ }^{9}$ alla quale si ispira, in quanto viene meno il riferimento alle sole fonti convenzionali relative ai diritti umani, inducendo a ritenere che non vi sia questo limite materiale, qualora il trattato abbia come oggetto la disciplina di regolazione del nucleo familiare.

Un cenno ancora per rilevare l'innovatività della Costituzione locale spetta anche al riferimento alla protezione ambientale, introdotto con una riforma del 2012 e che, oltre a richiamare la necessità di salvaguardare l'ambiente per la salute e il benessere dei cittadini, opera un riferimento alla necessità di agire in modo da non compromettere le necessità delle generazioni future. Si apprezza in questo inciso, l'accortezza di non aver utilizzato il termine "diritti" per i posteri — che è controverso in dottrina- ${ }^{10}$ ma più genericamente quello di "necessità", sancendo un obbligo di preservare e conservare le ricchezze naturali in senso transgenerazionale. Quest'ultimo riferimento è particolarmente rilevante perché costituisce una novità rispetto al testo federale.

Sempre nel tentativo di cogliere i profili più inediti del costituzionalismo locale, si può apprezzare che il legislatore di revisione di Puebla ha

\footnotetext{
8 Oltre a ciò si fa riferimento anche a "ordenamientos secundarios".

9 La disposizione è poi ripresa dall'art. 7 della Costituzione di Puebla. Sul tema in generale, Ortiz Ahlf, Loreta, Régimen interno e internacional de los tratados, México, Tirant lo Blanch, 2017.

10 Bifulco, Roberto, Diritto e generazioni future. Problemi giuridici della responsabilità intergenerazionale, Milano, Franco Angeli, 2013; Bifulco, Roberto e D'Aloia, Antonio, "Le generazioni future come nuovo paradigma del diritto costituzionale", in Bifulco, Roberto e D'Aloia, Antonio (a cura di), Un diritto per il futuro. Teorie e modelli dello sviluppo sostenibile e della responsabilità intergenerazionale, Napoli, Jovene, 2008, p. 177; Sikora, Richard I. e Barry, Brian M. (a cura di), Obligations to Future Generations, Filadelphia, Temple University Press, 1978; Rawls, John, A Theory of Justice, Oxford, Oxford University Press, 1971; Pisanò, Attilio, Diritti deumanizzati. Animali, ambiente, generazioni future, specie umana, Milano, Giuffrè, 2012; Cerutti, Furio, "Democrazia e/o generazioni future", Il Mulino, num. 3, 2012, pp. 389 e ss.
}

Esta obra está bajo una Licencia Creative Commons

Atribución-NoComercial-SinDerivar 4.0 Internacional, IIJ-UNAM. 
inteso rafforzare i legami dei rappresentanti politici sul territorio introducendo una disposizione che obbliga questi ultimi, durante la sospensione dei lavori del Congresso, a recarsi in visita nei distretti, al fine di essere edotti sulle condizioni dei propri cittadini e, in particolare, nei settori dell'istruzione pubblica, dell'industria, del commercio, dell'agricoltura e delle attività estrattive così da essere in grado di proporre misure idonee per superare condizioni di debolezza (art. 38).

Si tratta di una disposizione introdotta nel 1982 e di cui non vi è un corrispettivo nella Costituzione federale e che testimonia la volontà di avvicinare gli eletti alla comunità di riferimento; non si tratta di un'invocazione a coltivare il collegio ma di essere consapevoli delle condizioni del tessuto sociale in alcuni settori strategici per il paese.

Infine, un altro profilo in cui si manifesta una significativa originalità nella Costituzione di Puebla concerne gli strumenti di democrazia partecipativa, che sono declinati attraverso il referendum e il plebiscito. In questo ambito, il distacco con la Costituzione federale è particolarmente cospicuo, in quanto questa non prevede questi istituti di democrazia diretta o di partecipazione e, quindi, le entità federative, in assenza di divieti espliciti da parte di fonti costituzionali, hanno potuto cimentarsi in questo sforzo di inclusione democratica.

Il referendum abrogativo è disciplinato dagli artt. 68 e 69 e si segnala per alcuni aspetti peculiari. In primo luogo, emerge qualche incertezza sui limiti di ammissibilità, poiché possono essere sottoposte a referendum le leggi che posso essere rilevanti per l'ordine pubblico e per l'interesse sociale. E' di palmare evidenza che l'ampiezza e l'indeterminatezza degli enunciati linguistici possano far ricadere nell'istituto referendario qualsiasi atto legislativo, essendo quasi una prova diabolica argomentare quale legge non presenti un qualche interesse sociale. Peraltro, sono espressamente incluse le leggi elettorali, in quanto per questo specifico tipo di atto, viene vietata la celebrazione del referendum negli otto mesi precedenti le operazioni elettorali e durante le stesse. I limiti di ammissibilità espressi sono, invece, più definiti e si suddividono in primo luogo, fra quelli che riguardano fonti di rango superiore a quello ordinario, come le leggi di revisione costituzionale statale, le leggi organiche dei poteri dello Stato $^{11}$ e le leggi in attuazione di riforme costituzionali federali e in

11 C'è da chiedersi se altre leggi organiche possono essere escluse dal referendum in via estensiva. 
secondo luogo, fra quelli individuati ratione materiae e che includono le leggi tributarie. ${ }^{12}$ Resta impregiudicato un altro aspetto che la Costituzione non scioglie e cioè quale possa essere l'organo competente a verificare la legittimità e l'ammissibilità dei quesiti referendari. Da un punto di vista amministrativo, è presso l'Istituto elettorale di stato che gli elettori devono depositare le loro firme per richiedere il referendum ed è sempre l'organo amministrativo che gestisce tutte le operazioni elettorali. Dato il crescente rilievo che l'autorità elettorale assume nei singoli stati è da immaginare che anche il compito di verificare il rispetto delle norme procedurali sia affidato sempre all'Istituto, mentre, è meno chiaro quale potrà essere invece l'organo deputato a vagliare l'inammissibilità che, in genere, è uno di matrice giurisdizionale. ${ }^{13}$

Il quorum per la validità del referendum è indicato nel $40 \%$, che, comunque, rimane una soglia abbastanza alta in tutte quelle comunità politiche dove la partecipazione diretta stenta a vertebrarsi. Ma c'è un altro dato che rende difficile il perfezionamento di una procedura referendaria: si impone che la richiesta debba essere avanzata da almeno il $15 \%$ degli elettori, che allo stato attuale ammonterebbero a circa 626.000 persone, ${ }^{14}$ cifra che di per sé costituisce un filtro selettivo, soprattutto, poiché — come già detto- il tasso di mobilitazione politica non è alto.

Analoghe considerazioni possono essere svolte per il plebiscito, attraverso il quale i cittadini sono chiamati a esprimersi in via preventiva su atti o decisioni del governatore dello stato che siano rilevanti per l'ordine pubblico e l'interesse sociale (art. 85). L'iniziativa spetta al titolare del potere esecutivo (art. 79) e ai $2 / 3$ dei deputati del Congresso (art. 57). Su questo profilo, non possiamo esimerci dal sottolineare che qualora il procedimento sia attivato dall'organo rappresentativo, la consultazione può essere uno strumento di controllo dell'assemblea parlamentare nei confronti dell'Esecutivo, anche se non si tratta di un meccanismo che può essere attivato dall'opposizione parlamentare, visto che si richiede una maggioranza qualificata. Al contrario, se è il vertice della compagine governativa a proporre la consultazione, si può inferire che "la chiamata al popolo" si possa

\footnotetext{
12 Peraltro, la materia tributaria è esclusa anche dalle iniziative di legge popolare.

13 Su questo tema e anche più in generale sul tema degli istituti di democrazia partecipativa a livello comparato, Rodriquez, Simona, Rappresentanza democratica e strumenti di partecipazione, Napoli, Editoriale Scientifica, 2017.

14 Il dato è ricavato da: http://www.infoeleccionesmexico.com/informacion-numero-electores-por-estado-94.html.
} 
trasformare in un mezzo per consolidare il proprio indirizzo politico, piuttosto che uno strumento per implementare la partecipazione dei cittadini al procedimento decisionale.

Sono esclusi dal plebiscito gli atti che siano obbligatori per l'attuazione di leggi, quelli di organizzazione e funzionamento delle amministrazioni, quelli in materia tributaria e di spesa.

Nell'atto di convocazione dei comizi elettorali, devono essere esplicitate le conseguenze dell'adozione o meno dell'atto de quo e non si può non apprezzare lo sforzo che la Costituzione profonde nel tentativo di rendere consapevole la cittadinanza nelle sue scelte.

L'esito è vincolante nella misura in cui vi abbia partecipato il $40 \%$ degli iscritti alle liste elettorali e se una delle opzioni —o tutte quelle presentate- - abbia ricevuto l'assenso del 50\% dei voti.

Anche nella Costituzione di Puebla si tenta di separare il piano della democrazia diretta con quella rappresentativa e infatti è vietata l'indizione di plebiscito nell'anno delle elezioni e nei novanta giorni successivi alla chiusura delle urne; inoltre, al fine di evitare una proliferazione di momenti plebiscitari, è consentito indire una sola consultazione popolare all'anno.

\section{I DIRITTI DELLE POPOLAZIONI INDIGENE FRA GARANZIE E INATTUAZIONE}

La Costituzione federale espressamente rimanda al legislatore locale l'onere di introdurre una disciplina ad hoc sul tema del rispetto dei diritti delle comunità autoctone, pertanto, la questione indigena è una materia di competenza statale e non federale. ${ }^{15}$

D'altra parte, poiché le comunità native sul territorio messicano sono numerose, la scelta di affidare alle istituzioni delle entità federative il compito di adottare le misure più idonee sembra ampiamente condivisibile, in un'ottica di sussidiarietà verticale che inquadra nelle istituzioni più vicine alle comunità locali il locus più adeguato per intervenire efficacemente.

La dottrina individua tre fasi nell'incorporazione dei diritti degli indigeni nelle Costituzioni statali: la prima, che giunge fino al 1992, una che si situa nel lasso temporale dal 1992 al 2001e infine, la terza che è post 2001.

15 Cienfuegos Salgado, David, "Pueblos y comunidades indígenas en el constitucionalismo local", in Valadés, Diego e Carbonell, Miguel (coords.), El Estado constitucional contemporáneo, México, UNAM, Instituto de Investigaciones Jurídicas, 2006, pp. 135 e ss. 
Nel 1992, il legislatore di revisione federale introduce per la prima volta clausole che riconoscono il pluralismo culturale dello Stato messicano. Il riferimento è molto generico e solo in via interpretativa si ricava un obbligo di tutela delle culture indigene.

Il periodo che, invece, si snoda dal 1992 al 2001 è contrassegnato dagli Acuerdos de San Andrés Larraínzar, con i quali i rappresentanti dei popoli indigeni e il governo negoziano un patto che impegna il secondo a: riconoscere i popoli indigeni in Costituzione; ampliare la loro partecipazione politica; garantire il pieno accesso alla giustizia; promuovere le lingue e le culture autoctone; assicurare istruzione e formazione; sviluppare politiche per l'impiego; fornire protezione agli indigeni migranti; assicurare le necessità primarie. ${ }^{16}$ Gli Accordi non trovano un'immediata applicazione e per una riformulazione complessiva della costituzione di Puebla in senso indigenista, è necessario attendere il 2001. La legge di riforma costituzionale si pone l'obiettivo di intervenire in maniera organica e sistematica, riprendendo alcuni degli esiti degli Accordi di San Andrés: i contenuti degli artt. 4, 18, par. 6, 27, frac. VII, par. 2 e il 115, frac. III sono riformulati e ampliati nell'art. 2 della costituzione. ${ }^{17}$

Il contenuto di questa disposizione è molto articolato e investe molteplici ambiti: istruzione, salute, giustizia, politiche abitative, diritti linguistici, autorità tradizionali, diritti di partecipazione politica, regime proprietario, miglioramento dei servizi essenziali e delle infrastrutture nelle zone indigene e politiche sociali. È rilevante che l'ultimo comma dell'art. 2 richiama espressamente l'obbligo delle entità federative a dare attuazione ai principi espressi nella disposizione in esame nell'ambito delle proprie competenze.

16 Sámano Rentería, Miguel Ángel, "La lucha por el poder y los Acuerdos de San Andrés Larráinzar”, in Ordóñez Cifuentes, José Emilio (comp.), Balance y perspectivas del derecho social y los pueblos indios de Mesoamérica, México, UNAM, Instituto de Investigaciones Jurídicas, 1999, pp. 165 e ss.; Sámano Rentería, Miguel Ángel e Durand Alcántara, Carlos e Gómez González, Gerardo, "Los Acuerdos de San Andrés Larráinzar en el contexto de la Declaración de los Derechos de los Pueblos Americanos", disponibile in: www.juridicas.unam.mx, 2001; Rangel, Jesús Angel de la Torre, "Los Acuerdos de San Andrés, veinte años después: derecho insurgente”, Revista de Investigaciones Jurídicas, Escuela Libre de Derecho, núm. 40, 2016, pp. 169 e ss.

17 López Bárcenas, Francisco, "Los pueblos indígenas en las Constituciones de México”, Argumentos. Estudios Críticos de la Sociedad, núm. 82, septiembre-diciembre de 2016, pp. 181 e ss. 
Se il quadro temporale è corretto, si rileva che Puebla si posiziona fra quegli stati che, solo quando la disposizione federale sul riconoscimento del pluralismo culturale è divenuto precettiva, ha provveduto ad adottare norme in favore delle comunità indigene, a differenza di molti altri stati. ${ }^{18}$ La riforma è stata perfezionata nel dicembre $2004 \mathrm{e}$ in quel frangente, sono stati oggetto di nuova disciplina l'art. 11, che ha aggiunto il divieto di discriminazione per origine etnica o nazionale, l'art. 12, in cui si rinviene in capo allo Stato l'obbligo di proteggere i diritti dei popoli e delle comunità autoctone e che questa attività è da considerarsi di ordine pubblico e interesse sociale; infine, l'art. 13, che dichiara che lo stato di Puebla vanta una composizione "plural y multilingüistica" ed elenca i nomi dei principali gruppi etnici. ${ }^{19}$

L'attuazione della Costituzione federale da parte dello stato di Puebla può essere definito deficitaria. Il disposto legislativo federale di riferimento è assai ampio articolato e va a incidere in molteplici settori; al contrario, le indicazioni del legislatore di revisione statale si sono mostrate parche sul tema. Affermazione che, peraltro, sembra corroborata da una rapida disamina dei contenuti di altre carte fondamentali statali. Infatti, merita sottolineare l'originalità, e al contempo la rilevanza, dell'art. 13 della Costituzione dello stato di Chiapas, approvato il 13 giugno 1999, (ben prima della riforma federale del 2001), dove vengono anticipati alcuni temi incorporati nel testo federale come l'elezione delle proprie autorità tradizionali in conformità con gli usi e costumi, l'applicazione delle regole tradizionali nei procedimenti giurisdizionali in cui una delle parti è indigena, l'obbligo di fornire un traduttore nei giudizi, la previsione nei municipi a maggioranza indigena di meccanismi alternativi di risoluzione delle controversie basati su usi, costumi, tradizioni e valori culturali delle comunità native. Da segnalare è anche la Costituzione di Oaxaca, che in larga parte riprende i contenuti di quella del Chiapas ma che si distingue per la specifica attenzione riservata ai diritti politico-elettorali ricondotti agli usos y costumbres indígenas.

18 Gli stati che per primi hanno adottato diritti costituzionali espressi sono stati Guerrero (1987), Chiapas (1990), Oaxaca, (1990), Querétaro (1990), Hidalgo (1991). Nella seconda fase si annoverano: San Luis Potosí (1992), Sonora (1992), Veracruz (1993), Nayarit (1993 e poi 1998), Durango (1994), Jalisco (1994), Chihuahua (1994), Estado de México (1995), Campeche (1996), Quintana Roo (1997), Michoacán (1998). Cienfuegos Salgado, David, op. cit., p. 137.

19 Nahuas, Totonacas o Tutunakuj, Mixtecas o Nuu Savi, Tepehuas o Hamaispini, Otomíes o Hñähñü, Popolocas o N'guiva y Mazatecas o Ha shuta. 
Tuttavia, nonostante il ritardo nell'apertura alla tematica autoctona da parte dell'ordinamento costituzionale poblano, si segnalano alcuni profili di sicuro rilievo: le comunità indigene sono riconosciute come soggetti di diritto pubblico (come, peraltro, già indicato all'art. 2 della Costituzione federale) e viene invocata la necessità che siano sviluppate le forme interne di organizzazione sociale, culturale, politica ed economica. Particolare importanza assume il tema del sistema normativo indigeno: infatti, si prevede l'istituzione di autorità tradizionali, le quali possono dirimere le controversie interne applicando regole tradizionali, previa adozione di una legge che specifichi le modalità di riconoscimento delle loro decisioni; specularmente si afferma che, nei giudizi in cui le parti siano membri della comunità a titolo individuale o collettivo, il potere giudiziario debba tenere conto delle pratiche e delle consuetudini delle tribù, secondo quanto stabilito dalla legge.

L'osmosi fra l'ordinamento indigeno e quello ordinario risponde a un'esigenza fortemente rivendicata dai gruppi aborigeni, non solo a livello messicano ma anche internazionale. L'applicazione di regole tradizionali presuppone il fatto che le comunità indios costituiscano a tutti gli effetti dei veri e propri ordinamenti e che nel passato avessero esercitato una piena sovranità su quelle terre. Tali aspirazioni sono temperate dalla presenza di clausole limitative che intendono armonizzare le rivendicazioni indigeniste con i principi supremi dell'ordinamento messicano. ${ }^{20}$ In questa ottica, il riferimento è all'indicazione che le autorità tradizionali, che pure possono implementare una propria organizzazione sociale, economica, culturale e politica, devono agire rispettando il patto federale e la sovranità dello Stato. Queste precisazioni sono tese a escludere che il diritto all'autogoverno possa condurre a ipotesi secessionistiche e che possa essere rivendicato un pieno diritto all'autodeterminazione; il corpus di regole autoctone applicabili deve conformarsi ai principi generali della costituzione federale e statale con l'obbligo di non violare le garanzie dei diritti fondamentali e specificamente quelli riguardanti le donne. ${ }^{21}$

La Costituzione statale si diffonde, inoltre, su altri aspetti degni di menzione ed estremamente delicati come il controllo delle risorse naturali che

20 Rodríguez Martínez, Elí, "El artículo segundo constitucional y los conflictos de leyes en materia de derechos indígenas: problemas relativos al resurgimiento de los estatutos personales", in Carbonell, Miguel (coord.), Derecho constitucional, México, UNAM, Instituto de Investigaciones Jurídicas, 2004, pp. 1035 e ss.

21 Queste indicazioni sono estremamente tributarie della Costituzione federale. 
da sempre costituisce uno degli elementi di frizione fra le autorità autoctone e quelli statali, tuttavia, come in altri casi, i riferimenti non si discostano in maniera sensibile dagli enunciati della Costituzione federale e, soprattutto, non esprimono certo un favor verso le popolazioni aborigene; infatti, l'uso e lo sfruttamento delle terre è riconosciuto solo in via preferenziale agli aborigeni in conformità con il regime di proprietà individuale tutelato dalla costituzione.

Si richiama, inoltre, un generale dovere dello stato e dei municipi allo sviluppo delle lingue, culture, risorse, usi e costumi indigeni e in questo ambito si prefigura la necessità di costituire istituzioni e realizzare politiche che agiscano in maniera congiunta per dare impulso all'occupazione indigena, adeguare le politiche abitative ai fabbisogni delle tribù, promuovere l'educazione bilingue, interculturale, laica e differenziata, dare avvio a programmi culturali, sportivi e ricreativi, costruire infrastrutture e incorporare la medicina tradizionale nel sistema sanitario pubblico.

Particolarmente interessante - e in linea con le tendenze che emergono nel diritto comparato - è il riferimento alla necessità di realizzare meccanismi di consultazione idonei ed efficaci per consentire alle comunità autoctone di poter contribuire alla definizione dei piani statali e municipali di sviluppo, oltre che a determinare il contenuto delle misure legislative e amministrative che possono incidere sulle loro condizioni..$^{22}$ Purtroppo, né la massima fonte federale, né quella statale si spingono a prevedere un vero e proprio diritto alla consultazione degli interessati ogni qualvolta sia da definire il contenuto di un atto normativo che possa avere riflessi sulle condizioni di quelle popolazioni.

Siamo ben distanti, da quanto affermato dalla Corte Suprema canade$\mathrm{se}^{23}$ e cioè che sussista un "duty to consult" da parte delle autorità nel caso in cui queste debbano adottare delle misure che incidano sullo status delle

22 Tapia Gutiérrez, Asier, "La consulta previa y la debida diligencia: similitudes y diferencias de dos instrumentos para un modelo de desarrollo y la defensa de los derechos humanos", Derechos y Libertades, vol. 34, enero de 2016, pp. 235 e ss.

23 Cfr. Haida Nation v. British Columbia (Minister of Forests) [2004] 3 S. C. R.; Taku River Tlingit First Nation v. British Columbia (Project Assessment Director) [2004] 3. S.C.R. 2004; Mikisew Cree First Nation v. Canada (Minister of Canadian Heritage) [2005] 3 S. C. R. 388; Beckman v. Little Salmon/Carmacks First Nation [2010] 3 S. C. R. 103 e da ultimo Ktunaxa Nation v. British Columbia (Forests, Lands and Natural Resources) [2017] 2 S.C.R. 386. 
comunità e soprattutto sui territori ove risiedano. ${ }^{24} \mathrm{Va}$, nondimeno, avvertito che anche la Corte Interamericana dei Diritti Umani ha prefigurato un diritto per i nativi a essere consultati e, ove possibile, anche un obbligo per i governi di trovare un accordo..$^{25}$

Infine, ancora in ossequio al testo Costituzione federale, ci sono rimandi espliciti alla necessità di garantire $\mathrm{i}$ diritti delle popolazioni aborigene che sono stanziati fuori dai confini statali, oltre a che offrire ricovero e tutela a quegli indigeni che si trovino a qualsiasi titolo a transitare o risiedere nel territorio statale.

Volendo trarre delle conclusioni sulle disposizioni che riguardano la garanzia dei popoli nativi, ci troviamo costretti a ripetere che i contenuti dei disposti costituzionali sono mutuati in larghissima parte dal testo costituzionale federale, quasi a riproporne il testo integralmente. Sono, infatti, esigue le clausole innovative od originali come, per esempio, quella di procedere ad assunzioni preferenziali di indigeni per opere, programmi e azioni istituzionali.

24 Merita rilevare che la legislazione sui diritti delle identità culturali autoctone si caratterizza, in genere, per la specificità dell'iter legis, nel fatto che l'introduzione nell'ordinamento di norme riguardanti le tribù, è preceduta da apposite intese fra le autorità statali e rappresentanti delle diverse comunità autoctone, secondo un procedimento decisionale che ricalca quello dell'ordinamento internazionale, basato sul principio dell'unanimità piuttosto che su quello di maggioranza, proprio delle assemblee legislative. Gli esiti normativi delle relazioni fra livelli istituzionali e autorità indigene, sono sempre, quindi, anticipati da negoziati e questo sembra essere un dato costante e consolidato della storia fra gli autoctoni e le autorità canadesi, tanto da poter configurare una convenzione costituzionale in tal senso. Infatti, i trattati fra le comunità aborigene e i poteri pubblici rappresentano una fonte utilizzata in maniera diffusa per l'esatta definizione dei diritti spettanti alle comunità native. Vari elementi concorrono a ritenere che sia esistente una convenzione costituzionale in tal senso. Oltre al reiterato comportamento in tal senso e la più volte confermata volontà del governo di procedere in tale direzione, è la stessa Corte Suprema che in alcune sue più rilevanti pronunce sottolinea la necessità di ricomporre le contrapposizioni che possono sorgere nella società canadese con la negoziazione e la partecipazione dei soggetti coinvolti. È da aggiungere che l'obbligo di consultazione costituisce uno strumento di riconciliazione fra i nativi e la maggioranza bianca, Ritchie, Kaitlin, "Issues Associated with the Implementation of the Duty to Consult and Accommodate Aboriginal Peoples: Threathing the Goals of Reconciliation and Meaningful Consultation", University of British Columbia Law Review, vol. 46, 2013, pp. 397 e ss.; Newman, Dwight, Revisiting the Duty to Consult Aboriginal Peoples, Saskatoon, Purich, 2014.

25 Cfr., Saramaka People v. Suriname, Judgment of 28 November 2007 InterAmerican Court.

Esta obra está bajo una Licencia Creative Commons

Atribución-NoComercial-SinDerivar 4.0 Internacional, IIJ-UNAM. 
Al contrario, non tutte le prescrizioni previste dalla carta fondamentale federale hanno trovato spazio nel testo costituzionale statale, che, quindi, non ha colto tutte le potenzialità che ne potevano scaturire. A titolo esemplificativo, possiamo citare il caso dell'art. 115 par. VI della Costituzione federale che consente il coordinamento e l'associazione delle comunità indigene all'interno del municipio, di cui la Costituzione di Puebla non fa alcuna menzione.

Analogamente, il testo costituzionale di Puebla non sembra, invece, aver ripreso in maniera robusta i numerosi richiami alla tutela delle donne indigene. Come è noto, questo è un tema che a più riprese viene affrontato ogni qualvolta viene invocato il riconoscimento di diritti "speciali" per i popoli autoctoni. Il timore è che l'applicazione di regole tradizionali si converta nella costruzione di situazioni discriminatorie e marginalizzanti nei confronti delle donne. Al fine di scongiurare questa ipotesi, la carta federale in più disposizioni sottolinea come il riconoscimento dei diritti tradizionali non più affermarsi in violazione dei diritti delle donne. La suggestione è ripresa in maniera anastatica, laddove si prefigura l'applicazione dei sistemi normativi indigeni ${ }^{26}$ ma non sono tratti dal livello federale altri precetti, che richiamavano la necessità di favorire l'inclusione delle donne nei progetti di sviluppo, proteggere la loro salute, favorire i processi educativi e la loro partecipazione ai processi deliberativi della comunità (art. 2, B, frac. V) ovvero di porre particolare riguardo nella definizione delle politiche sociali nei confronti del genere femminile (art. 2o., B, frac. VIII).

La Costituzione di Puebla è silente anche sui diritti politici degli indigeni in generale: eppure il testo federale si esprime sul punto, facendo un riferimento, in primo luogo, al principio di parità di accesso ai pubblici uffici e, in secondo luogo, all'onere per gli stati di introdurre meccanismi atti a eleggere rappresentanti indigeni nei municipi. E' appena il caso di rammentare quanto sia strategica per rafforzare in generale i diritti delle popolazioni indigeni la rappresentanza politica, soprattutto in quegli enti che sono più vicini alla comunità sociale.

Tuttavia, nello stato di Puebla tale tematica non sembra essere nell'agenda legislativa, nonostante il richiamo echeggiato in Costituzione e l'esistenza di esempi virtuosi in tal senso, come lo stato di Oaxaca, che si è

26 Sia la Costituzione federale (art. 2o. par. A, frac. II) che la Costituzione di Puebla (art. 13, I, lett. d) richiamano la dignità e l'integrità delle donne. 
fatto promotore legislativamente della partecipazione indigena con modalità innovative. ${ }^{27}$

\section{ALCUNE NOTE CONCLUSIVE}

La Costituzione di Puebla si distingue per riprodurre in maniera anastatica le disposizioni della Costituzione federale e, pertanto, è complesso far emergere l'apporto originale dei "costituenti" statali, che nella maggior parte dei casi si sono limitati a incorporare le clausole federali. Tuttavia, in più di un'occasione, si può notare come il processo di riproduzione sia parziale, in quanto questa operazione di mimesi esclude alcuni disposti che, invece, potrebbero qualificare il testo positivamente.

Alcune di queste omissioni sono già state indicate nel contributo ma ne possiamo aggiungere di più significative. Ad esempio nella parte relativa al potere giudiziale, il sistema di nomina del Tribunal Superior de Justicia è ricalcato esattamente sulla procedura prevista a livello federale per i Magistrati alla Suprema Corte de Justicia de la Nación e cioè presentazione da parte del presidente della Repubblica di una terna di nomi al Senato, il quale dovrà poi dovrà procedere alla elezione; in Puebla, gli attori sono rispettivamente l'esecutivo e l'assemblea statale. Anche i requisiti per la nomina sono gli stessi, ${ }^{28}$ tuttavia, mentre la Costituzione federale del 1994 ha aggiunto all'art. 95, l'indicazione che preferibilmente i candidati alla nomina abbiano svolto funzioni giudiziarie o che abbiano avuto una carriera onorata e qualificata nell'ambito del diritto, questi elementi sono stati omessi dal legislatore locale di revisione, che, pure ha modificato l'art. 89 della Costituzione sul punto solo nel 2011.

E' chiaro che l'emendamento federale aggiuntivo ha come fine primario quello di qualificare in senso professionale la carica di magistrato dell'alta Corte, obiettivo che non sembra prioritario per il legislatore statale. ${ }^{29} \mathrm{Si}$

27 Preme rilevare che l'esperienza di Oaxaca ha attirato anche molte critiche. Per una disamina sull'esperienza, cfr. González Oropeza, Manuel e Martínez Sánchez, Francisco, El derecho y la justicia en las elecciones de Oaxaca, México, Tribunal Electoral del Poder Judicial de la Federación, 2011.

28 Cittadinanza per nascita, avere almeno 35 anni, aver conseguito una laurea in giurisprudenza da almeno 10 anni; avere una buona reputazione e non aver subito condanne penali superiori a un anno.

29 Sulla descrizione del sistema giudiziario a livello statale: Gámiz Parral, M. N., 
tratta quindi di un processo imitativo della Costituzione federale ma che è al contempo selettivo.

Un'altra carenza che si staglia nitidamente riguarda l'assenza di un organo di garanzia circa il rispetto delle disposizioni costituzionali statali. Infatti, non viene indicato nel testo se il Tribunal Superior de Justicia sia competente a verificare la conformità delle leggi statali alla Costituzione locale e neppure nella ley orgánica del Poder Judicial si fa cenno alla questione e, infine, non è stato istituito alcun organo che possa vagliare la compatibilità degli atti legislativi né in via preventiva, né successiva. Pertanto, sembra di poter capire che gli eventuali contrasti possono essere sciolti solo in via politica. ${ }^{30}$

Infine, un breve commento può essere riservato al tema della revisione costituzionale. Il procedimento emendativo impone la votazione del disegno di legge con la maggioranza dei $2 / 3$ dei deputati presenti e il consenso della maggioranza dei municipi dello stato (art. 140). Possiamo ribadire che la procedura non si differenzia nella sua impostazione dall'art. 135 della Costituzione federale che prevede la stessa maggioranza nel Congresso e per le assemblee degli Stati. Lo schema della Costituzione statale riverbera quella federale, istituendo un parallelismo fra i municipi - considerati a livello locale come istituzioni fondative- e le entità federative. ${ }^{31}$ Il procedimento, quindi, non è particolarmente aggravato e attenua le caratteristiche della rigidità, in quanto la maggioranza relativa non sembra molto complessa da raggiungere e con ciò si spiega anche il gran numero di riforme che il testo ha subito. Tuttavia, assoggettare alla stessa amending formula qualsiasi tipo di riforma da quella più marginale a quella più incisiva rischia di non garantire cospicuamente la rigidità costituzionale, forse più opportunamente si potrebbe immaginare di introdurre procedu-

Derecho constitucional y administrativo de las entidades federativas, Ciudad de México, UNAM, Instituto de Investigaciones Jurídicas, 2000, pp. 315 e ss.

30 Enríquez Soto, Pedro Antonio, "Régimen constitucional de las entidades federativas", www.juridicas.unam.mx; Madero Estrada, José Miguel, "Inviolabilidad y reformas de las constituciones estatales. Nayarit y su Constitución”, https://biblio.juridicas.unam. $m x / b j v$ (2001); Arteaga Nava, Elisur, "La constitución local y su defensa. Elementos para una teoría del control de constitucionalidad”, in Ferrer MacGregor, Eduardo e Vega Hernández, Rodolfo (coords.), Justicia constitucional local, México, Fundap, 2003, p. 11.

31 Valencia Carmona, Salvador, "La defensa constitucional del municipio mexicano", Anuario iberoamericano de justicia constitucional, vol. 6, 2002, p. 517; Guillén López, Tonatiuh, "La larga marcha del municipio mexicano", in Esquivel, Gerardo e Ibarra Palafox, Francisco Alberto e Salazar Ugarte, Pedro (coords.), op. cit., vol. III, pp. 173 e ss. 
re di revisione aggravate a geometria variabile. In altre parole, possiamo immaginare che la modifica di interi capitoli possa essere operata solo con iter procedurali più aggravati rispetto a quello previsto per micro-riforme. D'altra parte, il diritto comparato, in relazione alle Costituzioni nazionali, mostra alcuni esempi di tal guisa, è il caso della Spagna all'art. 168 che prevede la possibilità di riforma totale della Costituzione ovvero l'art. 74 della Costituzione Sudafricana ovvero gli artt. 38-47 del Canadian Constitution Act, 1982. 